\title{
Meconial peritrophic matrix and meconial degradation in the biting midge, Culicoides variipennis (Coquillett) (Diptera: Ceratopogonidae)
}

This article was published in the following Dove Press journal:

Open Access Insect Physiology

9 February 201 I

Number of times this article has been viewed

\section{Luis Renato León Villalba' William S Romoser ${ }^{2}$ Lisa Patrican ${ }^{3}$}

'Medical Entomology Laboratory, Institute of Microbiology, School of Biological and Environmental Sciences, Universidad San Francisco de Quito, Cumbaya, Quito, Ecuador; ${ }^{2}$ Tropical Disease Institute, Department of Biomedical Sciences, College of Osteopathic Medicine, Ohio University, Athens, OH, USA; ${ }^{3}$ National Center for Medical Intelligence (NCMI), Infectious Disease Division, Fort Detrick, MD, USA
Correspondence: William S Romoser Department of Biomedical Sciences, College of Osteopathic Medicine, Ohio University, Athens,

OH 4570I, USA

$\mathrm{Tel}+\mathrm{I} 7405923417$

Fax +I 7405972778

Email wromoser@gmail.com
Background: The peritrophic matrix (PM), a noncellular layer surrounding the food bolus in the gut, has been described in several invertebrate phyla, including Arthropoda. In arthropod vectors, the PM may be a barrier to pathogens in a meal. In mosquito pupae, a PM forms around the sloughed larval midgut epithelial cells (meconium), and evidence suggests this meconial PM (MPM) protects the developing adult midgut epithelium from microbes ingested during the larval stage. Given the probable protective function of MPM and the possibility that it exists in taxa beyond the Culicidae, we looked for MPM in a representative of a related dipteran family, Certatopogonidae (biting midges).

Methods: One hundred thirteen mature Culicoides variipennis (Diptera: Ceratopogonidae) larvae, pupae over the entire pupal stadium, and several adults were paraffin-sectioned, stained, and examined using light microscopy.

Results: Near the end of the larval stage, the midgut epithelium sloughs into the lumen forming the meconium and a new epithelium forms from regenerative cell proliferation. The meconium gradually histolyses as indicated by shrinkage, a staining reaction change from red to blue, and loss of cellular structure. A distinct MPM often forms and persists into the adult stage. Shortly after sloughing, blue staining material accumulates around the meconium and then disappears in correlation with MPM formation.

Conclusion: Our study supports the hypothesis that MPM occurs in taxa beyond the Culicidae, in this study the Ceratopogonidae, specifically in C. variipennis. As in mosquitoes, MPM occurrence is variable, and its formation may be induced in response to a chemotactic stimulus, possibly of microbial origin. As in mosquitoes, MPM in C. variipennis appears to protect the developing adult midgut by sequestering microorganisms remaining from the larval stage. Consistent with MPM induction and microbial sequestration, we saw in a few older pupae and new adults, brownish material, possibly microbial, within the confines of a distinct MPM.

Keywords: pupa, protection of developing midgut, microbial sequestration, diptera/microbe interaction

\section{Introduction}

The peritrophic membrane or matrix (PM), a chitinous, noncellular layer that surrounds the food bolus and separates it from the midgut epithelium, has been described in at least seven invertebrate phyla, including the arthropods. ${ }^{1-3}$ The PM is of particular interest in vector biology as a possible physical barrier between pathogens in a meal and the midgut epithelial cells. ${ }^{4}$

PM has been identified in the larval and/or adult stages of many insects, including mosquitoes. However, in mosquitoes, an additional PM is formed around the submit your manuscript | www.dovepress.com

Dovepress

DOI: 10.21 47/OAIP.SI3726
Open Access Insect Physiology 201 I:3 I-6

(C) 20 I I León Villalba et al, publisher and licensee Dove Medical Press Ltd. This is an Open Access article which permits unrestricted noncommercial use, provided the original work is properly cited. 
meconium during the pupal stage. ${ }^{5}$ The meconium is composed of degenerating larval midgut epithelial cells which begin to slough into the lumen late in the 4th larval stadium and complete this process early in the pupal stage. The sloughed larval midgut cells are replaced by basal regenerative cells which proliferate and form the new adult posterior midgut epithelium. Meconial PM (MPM) has been reported in seven mosquito species. ${ }^{5}$ In some cases, two MPMs form, the first (MPM1) early in the pupal stage and the second (MPM2) close to the time of adult emergence. ${ }^{5}$ MPM1 forms in temporal proximity to the first histological indicator of apolysis; that is, the appearance of molting fluid in the exuvial space. ${ }^{6}$ MPM appears first as precursor granules, which eventually coalesce into a continuous matrix surrounding the meconium. Ultrastructurally, mosquito MPM1 is similar to some layers in the larval PM, and MPM2 is very similar to the PM which forms around a blood meal in adult females. ${ }^{7}$ Moll et a $l^{8}$ provide evidence that MPMs confine microorganisms remaining from the larval stage and later facilitate their egestion, thereby preventing interference with adult midgut function. They also suggest that since molting fluid is ingested from the exuvial space during pupal-adult ecdysis, ${ }^{9}$ this hydrolytic enzymerich fluid contributes to the sterilization of the adult midgut. Given these findings in mosquitoes, it is possible that other insects that have generalist microplankton-feeding larvae form MPM, which functions in a similar way. Accordingly, we looked for MPM in pupae of Culicoides variipennis, another nematocerous dipteran that belongs to a different family than mosquitoes and has generalist feeding larvae.

The biting midge $C$. variipennis (Diptera: Ceratopogonidae) is the most important vector of bluetongue virus (BTV) ${ }^{10}$ in North America and a vector of epizootic hemorrhagic disease (EHD). ${ }^{11}$ Virus transmission occurs when infected females blood-feed on domestic animals such as sheep and cattle, causing disease and millions of dollars in losses to the livestock industry each year. As in other nematocerous Diptera, the minute, slender wormlike larvae of $C$. variipennis feed on microplankton in contrast to the adults, which are nectar (males and females) and blood (females) feeders. In the nonfeeding pupal stage, the body undergoes metamorphosis to the adult form, including a complete restructuring of the alimentary canal.

Our specific objectives were to: 1) determine whether MPM occurs in a taxon other than the family Culicidae, specifically in $C$. variipennis (family Ceratopogonidae, biting midges); 2) study the details of MPM formation and meconial degeneration; and 3) compare our results with previous studies of mosquitoes.

\section{Material and methods}

Larvae (C. variipennis, Medical Entomology Laboratory, Cornell University) were kept in plastic containers with gauze immersed in distilled water and fed TetraMin ${ }^{\circledR}$ (Tetra, Blacksburg, VA). They were held at a constant temperature of $29^{\circ} \mathrm{C} \pm 1{ }^{\circ} \mathrm{C}$ under a 16 -hour light, 8-hour dark regimen with fluorescent lighting. Adults were held in pint-size cardboard containers with fine mesh windows in the lids. To maintain high humidity, the containers were placed in plastic bags along with a water-saturated sponge.

A series of pupae of known hourly ages ( \pm 60 minutes) postpupation, representing the entire pupal stadium, and several 24- and 48-hour-old adults were killed and fixed using Bouin's solution. ${ }^{12}$ Specimens were dehydrated using a graded ethanol series, infiltrated with Paraplast ${ }^{\circledR}$ (SPI Supplies Division, Structure Probe, Inc.; West Chester, PA), embedded in Tissue-tek ${ }^{\circledR}$ paraffin blocks (Sakura Finetek USA, Inc.; Torrance, CA), and cut into $7 \mu \mathrm{m}$ serial sagittal sections with an $\mathrm{AO} 820^{\circledR}$ rotary microtome (American Optical Co., New York, NY). Sections were mounted on Fisherbrand ${ }^{\circledR}$ slides (Fisher Scientific; Pittsburgh, PA), stained using a simplified Azan trichrome technique, ${ }^{13}$ and studied with a Nikon Optiphot ${ }^{\circledR}$ light microscope (Nikon Instruments, Inc.; Melville, NY).

A total of 109 specimens were prepared: five larvae, 99 pupae (1-2 specimens for each hour postpupation), three 24-hour-old adults, and two 48-hour-old adults. Pupal stage duration in C. variipennis is approximately 49 hours. No attempt was made to separate females from males since no male-female differences were observed in mosquitoes. ${ }^{5}$

\section{Results \\ Degeneration of the meconium}

The larval midgut consists of an elongated tube comprising columnar epithelial cells and occupies most of the abdomen. It is delineated anteriorly and posteriorly by the cardia (outer layer of the proventriculus) and the pyloric valve, respectively. During the late larval stage (pharate pupa), the midgut epithelium begins to slough into the lumen forming a mass of degenerating cells, the meconium. The sloughed cells are replaced by the proliferation of small basal regenerative cells which form the adult posterior midgut.

Key changes in the meconium are shown in Figures 1A-E and presented in 4-hour age groups in Table 1. In the pharate pupa (Figure 1A), the meconium occupies most of the 


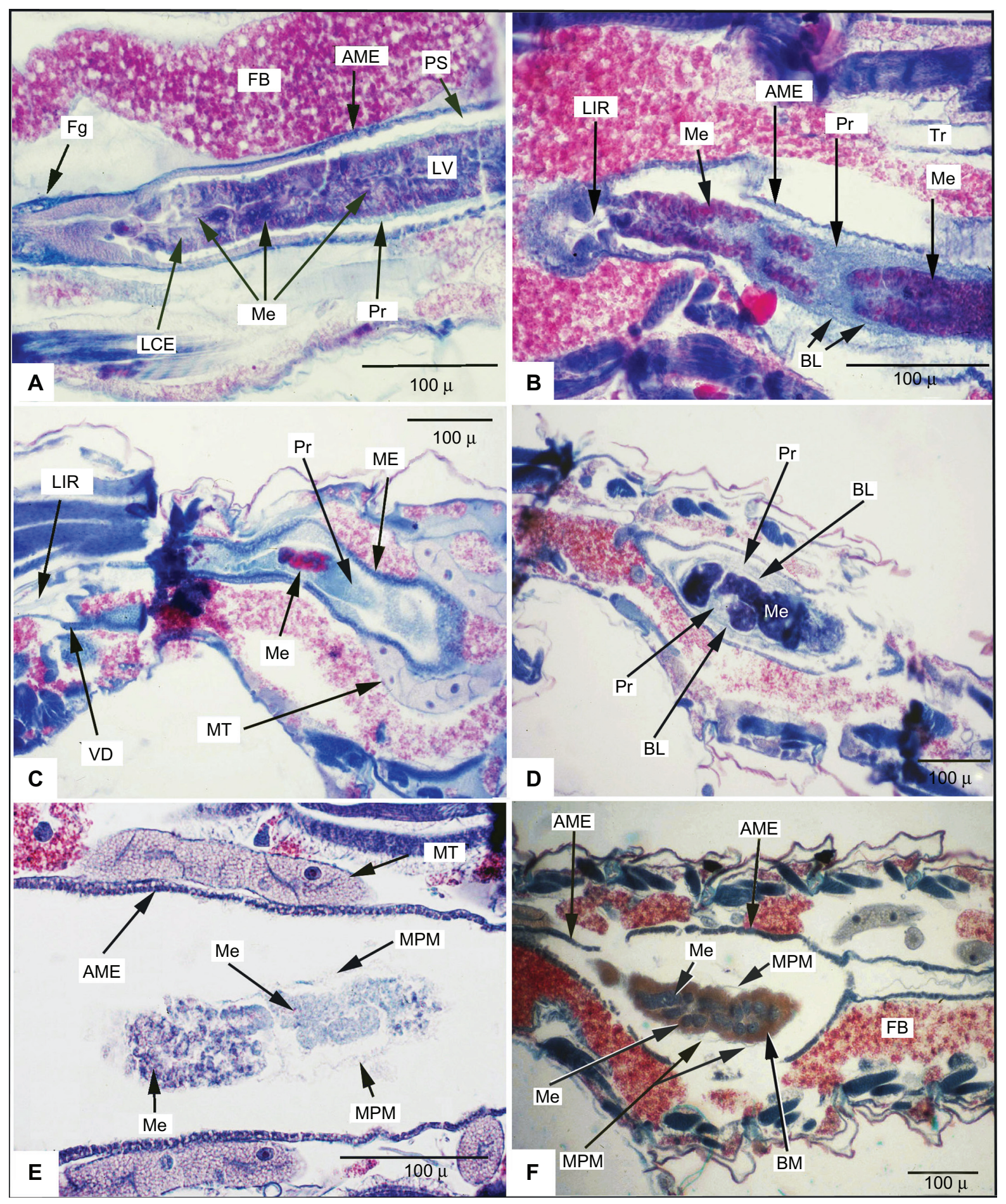

Figure I Sagittal sections of midgut pharate pupa A), 2-hour pupa B), 20-hour pupa C), 2I-hour pupa D), 35-hour pupa E), and 35-hour pupa showing "brown" material F). Abbreviations: AME, adult midgut epithelium; BL, blue line; BM, brown material; FB, fat body; Fg, foregut; LCE, larval cardial epithelium; LV, larval ventriculus; LIR, larval intimal remnant; Me, meconium; ME, midgut epithelium; MPM, meconial peritrophic matrix; MT, malpighian tubule; Pr, precursor material; PS, peritrophic space; Tr, trachea;VD, ventral diverticulum.

midgut lumen and is separated from the developing adult midgut epithelium by a thin peritrophic space. The degenerating meconial cells stain brownish purple with dark-stained nuclei. Early in the sloughing process, various parts of the larval midgut can still be identified (Figure 1A, laval cardial epithelium and larval ventriculus), and it is often possible to distinguish a remnant of the larval midgut lumen within the meconium. The meconium gradually degenerates, undergoes changes in shape and staining reaction, and cellular structural detail disappears. 
Table I Histological changes as a function of time postpupation

\begin{tabular}{|c|c|c|c|c|c|c|c|c|c|c|}
\hline Hours & $0-4$ & $5-9$ & $10-14$ & $15-19$ & $20-24$ & $25-29$ & $30-34$ & $35-39$ & $40-44$ & $45-49$ \\
\hline Cell detail lost & $\begin{array}{l}30^{\mathrm{a}} \\
(3 / 10)^{\mathrm{b}}\end{array}$ & $\begin{array}{l}50 \\
(5 / 10)\end{array}$ & $\begin{array}{l}50 \\
(5 / 10)\end{array}$ & $\begin{array}{l}80 \\
(8 / 10)\end{array}$ & $\begin{array}{l}100 \\
(10 / 10)\end{array}$ & $\begin{array}{l}100 \\
(10 / 10)\end{array}$ & $\begin{array}{l}100 \\
(10 / 10)\end{array}$ & $\begin{array}{l}100 \\
(9 / 9)\end{array}$ & $\begin{array}{l}100 \\
(10 / 10)\end{array}$ & $\begin{array}{l}100 \\
(10 / 10)\end{array}$ \\
\hline Meconium red & $\begin{array}{l}60 \\
(6 / 10)\end{array}$ & $\begin{array}{l}100 \\
(10 / 10)\end{array}$ & $\begin{array}{l}100 \\
(10 / 10)\end{array}$ & $\begin{array}{l}90 \\
(9 / 10)\end{array}$ & $\begin{array}{l}50 \\
(5 / 10)\end{array}$ & $\begin{array}{l}0 \\
(0 / 10)\end{array}$ & $\begin{array}{l}0 \\
(0 / 10)\end{array}$ & $\begin{array}{l}0 \\
(0 / 9)\end{array}$ & $\begin{array}{l}0 \\
(0 / 10)\end{array}$ & $\begin{array}{l}0 \\
(0 / 10)\end{array}$ \\
\hline Meconium blue & $\begin{array}{l}0 \\
(0 / 10)\end{array}$ & $\begin{array}{l}0 \\
(0 / 10)\end{array}$ & $\begin{array}{l}0 \\
(0 / 10)\end{array}$ & $\begin{array}{l}50 \\
(5 / 10)\end{array}$ & $\begin{array}{l}40 \\
(4 / 10)\end{array}$ & $\begin{array}{l}100 \\
(10 / 10)\end{array}$ & $\begin{array}{l}100 \\
(10 / 10)\end{array}$ & $\begin{array}{l}100 \\
(9 / 9)\end{array}$ & $\begin{array}{l}100 \\
(10 / 10)\end{array}$ & $\begin{array}{l}100 \\
(10 / 10)\end{array}$ \\
\hline $\begin{array}{l}\text { Blue-staining } \\
\text { and thin lines }\end{array}$ & $\begin{array}{l}100 \\
(10 / 10)\end{array}$ & $\begin{array}{l}100 \\
(10 / 10)\end{array}$ & $\begin{array}{l}100 \\
(10 / 10)\end{array}$ & $\begin{array}{l}100 \\
(10 / 10)\end{array}$ & $\begin{array}{l}100 \\
(10 / 10)\end{array}$ & $\begin{array}{l}40 \\
(4 / 10)\end{array}$ & $\begin{array}{l}20 \\
(2 / 10)\end{array}$ & $\begin{array}{l}0 \\
(0 / 9)\end{array}$ & $\begin{array}{l}0 \\
(0 / 10)\end{array}$ & $\begin{array}{l}0 \\
(0 / 10)\end{array}$ \\
\hline MPM present & $\begin{array}{l}0 \\
(0 / 10)\end{array}$ & $\begin{array}{l}0 \\
(0 / 10)\end{array}$ & $\begin{array}{l}0 \\
(0 / 10)\end{array}$ & $\begin{array}{l}0 \\
(0 / 10)\end{array}$ & $\begin{array}{l}0 \\
(0 / 10)\end{array}$ & $\begin{array}{l}90 \\
(9 / 10)\end{array}$ & $\begin{array}{l}100 \\
(10 / 10)\end{array}$ & $\begin{array}{l}77.8 \\
(7 / 9)\end{array}$ & $\begin{array}{l}20 \\
(2 / 10)\end{array}$ & $\begin{array}{l}40 \\
(4 / 10)\end{array}$ \\
\hline
\end{tabular}

Notes: aPercent; ${ }^{b}$ Number showing characteristic/number examined.

Abbreviation: MPM, meconial peritrophic matrix.

In $60 \%(6 / 10)$ of the $0-4$-hour group of the pupae the meconial cells stained red, and 30\% (3/10) had lost cellular structural detail. Using the red staining reaction as indicative of histolysis, degradation of the meconium began in the anterior region, initially in the portion corresponding to larval cardial cells, and moved posteriorly. In the 5-9-hour and 10-14-hour age groups, $100 \%$ (20/20) of the specimens showed a partially red stained meconium, and 50\% (10/20) had lost cellular structural detail. This process continued, and in the 20-24-hour group (Figure 1C and Table 1), all cellular structure had disappeared, and the red-staining reaction had been replaced by a blue-staining reaction in $40 \%(4 / 10)$ of the specimens (Figure 1D and Table 1).

\section{MPM formation}

Late in the larval stage, after the larval midgut epithelium had begun to slough into the lumen, blue-staining granular material appeared around the meconium (Figure 1A, precursor material and Table 1). This material gradually became more abundant (Figure 1B, precursor material and Table 1), forming a dense homogenous mass with scattered thin, denser blue lines around the meconium and eventually occupied a large portion of the peritrophic space. In the age groups preceding the 25-29-hour group (Figures 1B-1D and Table 1), blue-staining material with darker lines was present in $100 \%$ (50/50) of the specimens examined, decreased to $40 \%(4 / 10)$ in the 25-29-hour group, to $20 \%(2 / 10)$ in the $30-34$-hour group, and to $0 \%(0 / 9)$ in the 35-39-hour group. The gradual disappearance of the blue-staining material was correlated with the appearance of distinct MPM (Figures 1D and 1E, MPM and Table 1).

In the 25-29-hour group, a thin distinct MPM was observed in $90 \%$ (9/10) of the specimens, and the meconium had become reduced to a small, folded tubular mass of blue-staining granules. As the meconium continued to shrink, the anterior and posterior extremities of MPM collapsed into anterior and posterior filaments where all meconial material had been digested. In the 30-34-hour age group, a partially formed MPM was observed in 100\% (10/10) of the specimens, and the earliest observation of a fully formed MPM was in the 35-39-hour group (Figure 1E and Table 1). No precursor material was observed in pupae older than 33 hours postpupation. In the 35-39-hour group, MPM was observed in $77.8 \%(7 / 9)$ of the specimens, $20 \%(2 / 10)$ in the 40-44-hour group, and 40\% (4/10) in the 45-49-hour group. After adult emergence, a thin MPM was observed in two out of three adults examined. In one specimen, a distinct MPM was observed surrounding the almost nonexistent blue remnant of meconium and forming long anterior and posterior filaments in the lumen of the midgut.

In three of the older pupa/adult specimens, the bluestaining meconial remnants were surrounded by a dense brownish mass of material which occupied part of the peritrophic space (Figure 1F, brown material). This material was completely enveloped by a very distinct MPM, and in one specimen, the MPM was perforated during histological preparation, and the brownish material had leaked into the peritrophic space.

\section{Discussion}

Meconial peritrophic membranes have been described in seven mosquito species including the genera Aedes, Anopheles, Culex, and Culiseta. ${ }^{5,7}$ Our study supports the hypothesis that MPM occurs in a taxon beyond the Culicidae; that is, in a representative of different nematocerous dipteran family, C. variipennis.

In C. variipennis, all pupae examined formed a meconium which degenerated during the pupal stage and the residue of which remained in the midgut lumen until after adult eclosion. This is identical to what occurs in mosquitoes. ${ }^{7}$ A red-staining 
reaction coincides with meconial histolysis, as indicated by shrinkage and loss of cellular detail. Histolysis appears to begin anteriorly and proceed posteriorly, with complete disappearance of cellular detail by 24 hours postpupation, and after which time the meconium displays a blue-staining reaction. Such an anterior to posterior degeneration has also been described in mosquitoes. ${ }^{7}$

In the late phase of meconial degeneration (after 25 hours), anterior and posterior filaments of collapsed MPM were seen as the meconium continued to shrink. As in mosquitoes, meconial shrinkage is assumed to be associated with the assimilation of the breakdown products of histolysis.

A distinct MPM usually develops around the meconium during the pupal stage of $C$. variipennis and often remains until after adult emergence. In C. variipennis pupae, we observed an accumulation of blue-staining granules formed into a dense homogeneous mass. Eventually these granules were seen to break apart and gradually disappear from the midgut lumen. This disappearance immediately preceded MPM formation. This observation is consistent with the interpretation of the blue-staining granules as MPM precursor material, as described in mosquitoes. ${ }^{7}$

Blue-staining lines of varying density were often evident on the surface of the meconium and/or within the bluestaining mass. We interpret these as lines of precursor material, elements of which tend to assemble and break apart until the time of resorption by the developing adult midgut epithelial cells and the final deposition of a continuous layer of MPM. The accumulation of likely MPM precursor material in both $C$. variipennis and mosquitoes is similar to that observed in the development of the esophageal diverticula in mosquitoes, in which blue-staining material that will eventually be incorporated into the foregut intima within the diverticula gradually accumulates in the lumen and then disappears, followed closely by the appearance of the intima as a continuous sheet. ${ }^{12,14}$ Romoser and Venard ${ }^{14}$ suggest that this gradual deposition of precursor material in the lumen of the developing diverticula serves as an extracellular storage mechanism facilitating the accumulation of precursor material sufficient to provide intimal cover for the greatly increased luminal surfaces of the fully differentiated diverticula. The formation of MPM in mosquitoes and in C. variipennis could be a similar extracellular accumulation and storage mechanism, facilitating accumulation of precursor material sufficient to completely cover the meconium.

In mosquitoes, two membranes, designated MPM1 and MPM2, have been reported; ${ }^{5}$ the first closely surrounding the surface of the meconium and the second surrounding the first one, but more loosely. In C. variipennis, we observed only a single MPM in the pupal/early adult stages. This MPM formed after 25 hours postpupation and was seen in the lumen of the gut after adult eclosion typically as a thin blue line surrounding the tubular mass of degenerating meconium. The histological appearance of this MPM, and the timing of its formation, indicate that it corresponds to MPM1 in mosquitoes.

Differences in the timing of formation of MPM1 have been reported in mosquitoes. In Aedes aegypti, Anopheles stephensi, and Culex pipiens, MPM1 is first observed in the 15-24-hour, 8-12-hour, and 8-12-hour groups, respectively. ${ }^{5}$ In $C$. variipennis, a distinct, fully formed MPM was not seen prior to 25 hours.

Prior studies of mosquitoes have revealed variation in the occurrence of MPMs. Romoser et al ${ }^{5}$ found MPM in $24.3 \%$ of Ae. triseriatus and from $21.9 \%$ to $77.1 \%$ of Ae. aegypti. This variation in MPM production raises the interesting possibility that MPM formation may be inducible in response to some kind of chemical stimulus, perhaps of microbial origin.

In C. variipennis, we found MPM present in 65.3\% (32/49) of the specimens older than 25 hours postpupation. However, the percentage varied within age groups. From 25 to 39 hours, MPM was observed in $89.6 \%(26 / 29)$ of the specimens, but decreased to $30 \%(6 / 20)$ after 40 hours postpupation. There are at least three possible, not necessarily mutually exclusive, explanations: 1) MPM is not always present, and its formation is inducible; 2) MPM is sometimes destroyed during preparation for microscopy; and 3) MPM sometimes degenerates before adult eclosion. The first explanation is consistent with observations in adult mosquitoes in which MPM is not always found ${ }^{5}$ and also consistent with the possible inducibility of MPM formation.

Romoser et $\mathrm{al}^{5}$ hypothesize that MPM protects the developing adult midgut epithelium by sequestering microorganisms present from the larval stage, and later facilitates their egestion in recently emerged adults. Moll et al ${ }^{8}$ provided support for this hypothesis when they found that in recently emerged Culex mosquitoes, bacterial counts were significantly higher in the MPM-encased meconium than in the peritrophic space. Further, they found a decrease in the number of bacteria in the larval gut after the last defecation before pupation, and then a gradual increase during the pupal stage. However very few bacteria were found in recently emerged adult mosquito midguts, correlating with egestion of the MPM and contents.

In three different specimens, we observed brownish material around the blue-staining meconium and encased by a distinct MPM. This is consistent with the idea that MPM 
forms a barrier between its contents and the peritrophic space. The origin of the brownish material is not known; however, it is likely to be associated with microorganisms ingested during the larval stage. It is, therefore, logical to hypothesize a microbial sequestering function of the MPM in the biting midges as well as its possible inducibility in response to a microbial stimulus.

Having found MPM in two nematocerous dipteran families, it is plausible to hypothesize the presence of MPMs among other holometabolous insect groups that have generalist feeding larvae and specialist feeding adults. On this basis, further investigation of MPM, a structure of potentially wide significance within the class Insecta, is warranted.

\section{Acknowledgments}

J Spielholz of the Diagnostic Laboratory, College of Veterinary Medicine, Cornell University provided the Culicoides variipennis larvae.

\section{Disclosure}

The authors disclose no conflicts of interest in this work.

\section{References}

1. Hegedus D, Erlandson M, Gillott M, Toprak U. New insights into peritrophic matrix synthesis, architecture, and function. Annu Rev Entomol. 2009;54:285-302.

2. Peters W. Peritrophic membranes. In: Bradshaw SD, Randall DJ, editors. Zoophysiology. Volume 30. Darmstadt, Germany: Springer-Verlag; 1992.
3. Spence KD. Structure and physiology of the peritrophic membrane. In: Binnington K, Retnakaran A, editors. Physiology of the Insect Epidermis. Clayton, Australia: CSIRO; 1991:77-93.

4. Jacobs-Lorena M, Oo MM. The peritrophic matrix of insects. In: Beaty BJ, Marquardt WC, editors. The Biology of Disease Vectors. Niwot, CO: University of Colorado Press; 1996:318-332.

5. Romoser WS, Moll RM, Moncayo AC, Lerdthusnee K. The occurrence and fate of the meconium and meconial peritrophic membranes in pupal and adult mosquitoes (Diptera: Culicidae). J Med Entomol. 2000; 37(6):893-896.

6. Walker E, Romoser WS. Early events in the pupal-adult molt of Aedes triseriatus (Diptera: Culicidae): spatial and temporal considerations. Ann Entomol Soc Amer. 1982;75(4):395-399.

7. Moncayo AC, Lerdthusnee K, Leon R, Moll RM, Romoser W. Meconial peritrophic membrane structure, formation and meconial degeneration in mosquito pupae/pharate adults: histological and ultrastructural aspects. J Med Entomol. 2005;42(6):939-944.

8. Moll RM, Romoser WS, Modrzakowski MC, Moncayo AC, Lerdthusnee K. Meconial peritrophic membranes and the fate of midgut bacteria during mosquito (Diptera: Culicidae) metamorphosis. $J$ Med Entomol. 2001;38(1):29-32.

9. Walker MC, Romoser WS. The origin and movement of gas during adult emergence in Aedes aegypti: an hypothesis. J Am Mosq Control Assn. 1987;3(3):429-432.

10. Tabachnick WJ. Culicoides variipennis and bluetongue-virus epidemiology in the United States. Annu Rev Entomol. 1996;41:23-43.

11. Wilson WC. Detection of epizootic hemorrhagic disease virus in Culicoides variipennis (Diptera: Ceratopogonidae). J Med Entomol. 1991;28(5):742-744.

12. Romoser WS, Venard CE. The development of the ventral oesophageal diverticulum in Aedes triseriatus (Diptera:Culicidae). Ann Entomol Soc Amer. 1966;59(3):484-489.

13. Hubschman JH. A simplified Azan process well suited for crustacean tissue. Stain Technol. 1962;37:39-40.

14. Romoser WS, Venard CE. The development of the dorsal oesophageal diverticula in Aedes triseriatus (Diptera: Culicidae). Ann Entomol Soc Amer. 1967;60(3):617-623.
Open Access Insect Physiology

\section{Publish your work in this journal}

Open Access Insect Physiology is an international, peer-reviewed, open access journal publishing original research, reports, reviews and commentaries on all areas of insect physiology. The manuscript management system is completely online and includes a very quick and fair peer-review system, which is all easy to use.

\section{Dovepress}

Visit http://www.dovepress.com/testimonials.php to read real quotes from published authors. 